\title{
Detecting differential growth of microbial populations with Gaussian process regression
}

\author{
Peter D. Tonner, ${ }^{1,2}$ Cynthia L. Darnell, ${ }^{2}$ Barbara E. Engelhardt, ${ }^{3}$ and Amy K. Schmid ${ }^{1,2}$ \\ ${ }^{1}$ Program in Computational Biology and Bioinformatics, Duke University, Durham, North Carolina 27708, USA; ${ }^{2}$ Biology \\ Department, Duke University, Durham, North Carolina 27708, USA; ${ }^{3}$ Computer Science Department, Center for Statistics \\ and Machine Learning, Princeton University, Princeton, New Jersey 08540, USA
}

\begin{abstract}
Microbial growth curves are used to study differential effects of media, genetics, and stress on microbial population growth. Consequently, many modeling frameworks exist to capture microbial population growth measurements. However, current models are designed to quantify growth under conditions for which growth has a specific functional form. Extensions to these models are required to quantify the effects of perturbations, which often exhibit nonstandard growth curves. Rather than assume specific functional forms for experimental perturbations, we developed a general and robust model of microbial population growth curves using Gaussian process (GP) regression. GP regression modeling of high-resolution time-series growth data enables accurate quantification of population growth and allows explicit control of effects from other covariates such as genetic background. This framework substantially outperforms commonly used microbial population growth models, particularly when modeling growth data from environmentally stressed populations. We apply the GP growth model and develop statistical tests to quantify the differential effects of environmental perturbations on microbial growth across a large compendium of genotypes in archaea and yeast. This method accurately identifies known transcriptional regulators and implicates novel regulators of growth under standard and stress conditions in the model archaeal organism Halobacterium salinarum. For yeast, our method correctly identifies known phenotypes for a diversity of genetic backgrounds under cyclohexamide stress and also detects previously unidentified oxidative stress sensitivity across a subset of strains. Together, these results demonstrate that the GP models are interpretable, recapitulating biological knowledge of growth response while providing new insights into the relevant parameters affecting microbial population growth.
\end{abstract}

[Supplemental material is available for this article.]

Quantification and prediction of microbial growth is a central challenge relevant to industrial production of value-added chemicals, food safety, and microbe-environment interactions (McKellar and Lu 2003; Ross and Dalgaard 2003; Nichols et al. 2011; Lewis et al. 2012). Parametric models of microbial population growth assume a sigmoid growth function with three characteristic growth phases captured by three parameters: lag phase time (lag phase; $\lambda$ ), during which no growth occurs; maximum growth rate during logarithmic growth (log phase; $\mu_{\max }$ ), a phase of rapid growth; and asymptotic carrying capacity (stationary phase; $A$ ), reached when nutrients are exhausted in stationary phase (Monod 1949; Zwietering et al. 1990; Baranyi and Roberts 1995; Egli 2009). Another quantification of growth is the area under the growth curve (AUC), also known as growth potential (Todor et al. 2014).

Microbial populations encounter shifts away from optimum growth conditions in their environment that require adaptation in order to survive. These shifts, generally referred to as stress conditions, include reactive oxygen species (ROS) accumulation, temperature variation, and osmotic fluctuation. These conditions chemically damage or denature macromolecules such as proteins, nucleic acids, and lipids, compromising cellular viability (Imlay 2003; Kühn and Klipp 2012; Verghese et al. 2012). During stress response, the cell state changes from a growth-centric to a survivalcentric configuration in which the transcriptional and translational programs are redirected to regulate alternative pathways that re-

Corresponding authors: bee@princeton.edu, amy.schmid@duke.edu Article published online before print. Article, supplemental material, and publication date are at http://www.genome.org/cgi/doi/10.1101/gr.210286.116. Freely available online through the Genome Research Open Access option. pair damage and restore homeostasis (Lu et al. 2009). When stress is severe, the repair program becomes overwhelmed. In this case, the population growth rate observed by optical density (OD) decreases, plateaus, and may even become negative upon cell lysis.

Existing methods used to model and predict microbial population growth from time-series measurements are parametric functions known as primary or secondary models (McKellar and Lu 2003; Ross and Dalgaard 2003; Peleg and Corradini 2011). Primary models are used to fit data from a population growing on a single main nutrient source (e.g., sugar carbon source) and often assume a sigmoid growth function. This functional assumption leads to inaccurate fits for growth data that do not have a characteristic sigmoid growth function (Sekse et al. 2012; Palacios et al. 2014). Secondary models were developed to incorporate additional parameters affecting growth and to capture stress effects (Peleg and Corradini 2011). The significance of differential growth across conditions can be quantified through statistical hypothesis testing (Gommers et al. 1988). However, incorporating condition-specific deviations to the sigmoidal growth function requires a priori knowledge of how stress perturbations affect growth. For example, a common assumption is that population growth rate follows an Arrhenius equation in response to temperature changes (Barsa et al. 2012). As an alternative to parametric models of population growth, nonparametric models have been developed (di Sciascio and Amicarelli 2008; Cao et al. 2010; Palacios et al. 2014); however, many of these models still depend upon parametric primary

(C) 2017 Tonner et al. This article, published in Genome Research, is available under a Creative Commons License (Attribution 4.0 International), as described at http://creativecommons.org/licenses/by/4.0/. 
models, parameters that require knowledge of the biological response to growth perturbations, or complicated fitting procedures of the nonparametric model, such as optimization of neural network weights (Palacios et al. 2014). Current models of microbial growth are therefore limited in their general application to novel microbial growth phenotypes.

Across all domains of life, stress response mechanisms at the level of gene transcription have been identified that regulate cellular protection and repair (Gasch et al. 2000; Bonneau et al. 2007; Fiebig et al. 2015). These regulatory programs are induced in response to stress conditions and protect cells exposed to one type of stress against other stressors (Jenkins et al. 1988; Lu et al. 2009). Conversely, cells also induce stress-specific responses to aid survival for a particular condition (Stephen et al. 1995; Zuber 2009). The hypersaline-adapted, or halophilic, archaeon Halobacterium salinarum is a model organism uniquely suited to study microbial stress response because it survives extremely high levels of ultraviolet (UV), ROS, heat shock, and other stressors in its desert salt lake niche (Ng et al. 2000; Oren 2008). As such, H. salinarum has been extensively studied as a model system for transcription regulatory network architecture and function in response to stress (Schmid et al. 2009, 2011; Todor et al. 2013, 2014; Tonner et al. 2015). A gene regulatory network inferred from transcriptomic data predicts that over 70 transcription factors (TFs) may regulate genes whose products adjust physiology and repair damage incurred by stress (Bonneau et al. 2007; Brooks et al. 2014). Network predictions have been used to characterize the full set of TF target genes and physiological roles of TFs that control the response to oxidative stress through RosR and AsnC (Sharma et al. 2012; Plaisier et al. 2014; Tonner et al. 2015), nutrient availability through TrmB (Schmid et al. 2009; Todor et al. 2013, 2014), metals through SirR (Kaur et al. 2006), iron homeostasis through Idr1 and Idr2 (Schmid et al. 2011), and copper response through VNG1179C (Kaur et al. 2006; Plaisier et al. 2014). Despite this knowledge, the cellular regulators of growth that respond to environmental perturbation remain understudied in $H$. salinarum and other archaeal species. In particular, the phenotypic impact of mutations to known TFs under alternate stress conditions-and the downstream effect of those mutations on the function of the global regulatory network-remains unclear for H. salinarum (Brooks et al. 2014) and many other understudied microorganisms (Yoon et al. 2011, 2013).

Here, we develop a Gaussian process (GP) regression model of microbial growth to overcome the limitations of parametric growth modeling. GPs are distributions on arbitrary functions, where any finite number of observations of the function are distributed as a multivariate normal (MVN) in a computationally tractable framework (Rasmussen and Williams 2006). Because GP regression fits an arbitrary functional form, it is able to model growth curves that deviate from a sigmoid form. We compare our model with several primary parametric models and establish the ability of GP regression to accurately model growth curves from $H$. salinarum under standard and stress treatments across genetic backgrounds. We show that the fitted GP model recovers biologically interpretable measures of microbial growth. We develop statistical tests of differential growth response between two experiments via data likelihoods computed from the fitted GP regression model. We call this model and testing framework Bayesian Growth Rate Effect Analysis and Test (B-GREAT). To demonstrate the general utility of B-GREAT, we applied it to yeast population growth data under diverse stress conditions and genetic backgrounds (Liti et al. 2009). In both $H$. salinarum and yeast applications, BGREAT recapitulates known differential growth phenotypes and enables discovery of novel phenotypes.

\section{Results}

We developed a GP regression model to capture population growth data and applied this model to data from seven $H$. salinarum TF mutants (Table 1). The growth of these strains was compared to the $\Delta u r a 3$ parent strain from which the mutants were derived under optimum nutrient conditions (referred to as "standard conditions") and chronic oxidative stress (see Methods). OD, which quantifies cell density, was measured using a high-throughput plate reader (Fig. 1; Supplemental Fig. S1). Population growth phenotypes were measured in a minimum of 12 samples per mutant per condition, sampled every $30 \mathrm{~min}$ over $48 \mathrm{~h}$ for a total of 12,720 data points (Supplemental Table S1). Chronic oxidative stress was induced by the addition of $0.333 \mathrm{mM}$ paraquat $(\mathrm{PQ})$ when the culture was inoculated. The growth rate of these TF strains under standard conditions during log phase has been tested previously (Kaur et al. 2006; Schmid et al. 2009, 2011; Sharma et al. 2012; Plaisier et al. 2014), but only the growth rates of TF knockout mutants $\Delta a s n C$, $\triangle$ trmB, and $\triangle$ rosR have been tested under PQ conditions (Table 1; Schmid et al. 2009; Sharma et al. 2012; Plaisier et al. 2014).

\section{GP regression model of microbial population growth}

In order to model the diverse growth phenotypes observed under both standard and oxidative stress conditions, a probabilistic model of population growth was constructed using GP regression (Fig. 1; Supplemental Fig. S1). GP regression is a Bayesian nonparametric model that describes the distribution over a function $f(x)$, of which any finite number of observations $\{x, f(x)\}$ have a MVN distribution (see Methods) (Rasmussen and Williams

Table 1. Strains used in this study and their known phenotypes and functions

\begin{tabular}{|c|c|c|c|c|c|}
\hline $\begin{array}{l}\text { Strain } \\
\text { name }\end{array}$ & Genotype & $\begin{array}{l}\text { Standard } \\
\text { growth }\end{array}$ & $\begin{array}{l}\text { Paraquat } \\
\text { exposure }\end{array}$ & Pathways regulated & Reference \\
\hline$\Delta u r a 3$ & Parent strain & $\mathrm{n} / \mathrm{a}$ & $\mathrm{n} / \mathrm{a}$ & $\mathrm{n} / \mathrm{a}$ & Peck et al. (2000) \\
\hline$\Delta t r m B$ & $\Delta u r a 3 \Delta$ trmB & Slow growth & none & Metabolism & Schmid et al. (2009); Todor et al. $(2013,2014)$ \\
\hline$\Delta \operatorname{ros} R$ & $\Delta u r a 3 \Delta \operatorname{ros} R$ & None & Slow growth & Oxidative stress repair & Sharma et al. (2012); Tonner et al. (2015) \\
\hline$\Delta i d r 1$ & $\Delta u r a 3 \Delta i d r 1$ & None & Not measured & Iron homeostasis & Schmid et al. (2011) \\
\hline$\Delta i d r 2$ & $\Delta u r a 3 \Delta i d r 2$ & None & Not measured & Iron homeostasis & Schmid et al. (2011) \\
\hline$\Delta \operatorname{sir} R$ & $\Delta$ ura3 $\Delta$ sirR & None & Not measured & Manganese uptake & Kaur et al. (2006) \\
\hline$\Delta V N G 1179 C$ & $\Delta$ ura3 $\triangle$ VNG1179C & None & Not measured & Copper uptake & Kaur et al. (2006) \\
\hline$\Delta a s n C$ & $\Delta$ ura 3 asnC & Slow growth & Slow growth & Oxidative stress repair & Plaisier et al. (2014) \\
\hline
\end{tabular}

All phenotypes were previously quantified only in log phase. Yeast strains analyzed are as previously described (Liti et al. 2009). 

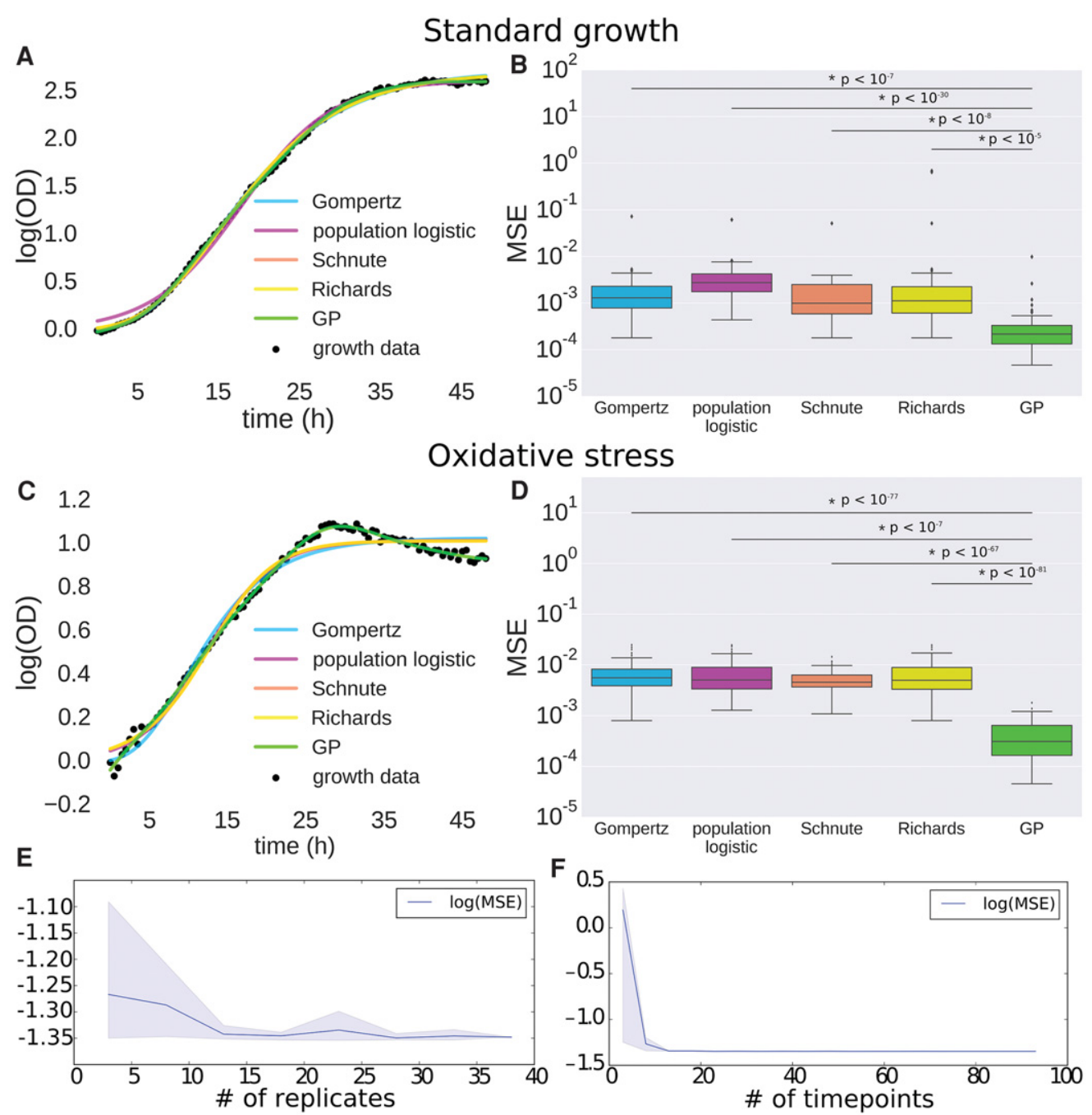

Figure 1. Gaussian process (GP) regression outperforms primary growth models. (A) Comparison of GP regression and primary growth models (Gompertz, population logistic, Schnute, and Richards) on microbial growth data under standard conditions. (B) Logarithm of mean squared error (MSE) for primary growth models compared with GP regression on microbial population growth under standard conditions. Bars with an asterisk indicate a significant difference between GP MSE and primary growth model MSE as determined by a one-sided $t$-test. $P$-values of the significance are indicated above the bars. (C) Comparison of GP regression and primary growth models on microbial growth data under oxidative stress. (D) Logarithm of MSE for primary growth models compared with GP regression on microbial population growth under oxidative stress. Bars with asterisks as in $B$. ( $E, F)$ Measure of MSE as a function of the number of replicates $(E)$ and of time points $(F)$ for GP regression. Solid lines represent mean MSE, and shaded regions represent empirical $90 \%$ confidence regions calculated from three random samplings of data at each number of replicates or time points.

2006). The GP model is described by its prior mean and covariance functions $\left(\mu(x)\right.$ and $\kappa\left(x, x^{\prime}\right)$, respectively). In this study, prior mean $\mu(x)$ was set to zero, as is standard (Rasmussen and Williams 2006). The kernel function was set to a radial basis function (RBF), $\kappa\left(x, x^{\prime}\right)$, defining the covariance matrix of this MVN distribution.

GP regression places a prior on all arbitrary functions mapping time to $\mathrm{OD}$, where the kernel function and parameterization encourage a specific smoothness of the function. Independent and identically distributed (IID) Gaussian noise with mean zero and variance $\sigma^{2}$ is assumed in each function observation $y=f(x)+$ $N\left(0, \sigma^{2}\right)$. Estimating the parameters of a GP regression model on microbial growth data is performed by maximizing the data likelihood with respect to the kernel function parameters (Rasmussen and Williams 2006). We refer to our model (and associated tests, described below) as Bayesian Growth Rate Effect Analysis and Test (B-GREAT).

\section{Evaluating kernel function choice for GP growth modeling}

In order to ensure that our choice of RBF kernel function accurately represented the data, we tested the use of Matérn and linear kernel functions compared with the RBF kernel function. Matern kernels are stationary, like RBF kernels, and model the covariance of data points as a function of their distance in $x$. Linear kernels are of the form $k(x, y)=\sum_{i=1}^{p} \sigma_{i} x_{i} y_{i}$, and the covariance increases with the magnitude of the covariates (Rasmussen and Williams 2006). The GP model with each of the three kernels was used to fit growth data for the $\Delta u r a 3$ parent strain under standard conditions. Model fits were assessed by the Bayesian information criterion (BIC) (Neath and Cavanaugh 2012). GPs with Matérn and RBF kernels have lower BIC scores than those with linear kernels, indicating that GP models with these kernels are more likely than those

\section{Genome Research}

www.genome.org 
with a linear kernel (Supplemental Fig. S2; Neath and Cavanaugh 2012). From this, we conclude that our use of RBF kernel functions is sufficient for these data.

\section{B-GREAT outperforms primary growth models}

B-GREAT was used to fit time-series growth data from $H$. salinarum $\Delta u r a 3$ parent strain populations under both standard and oxidative stress conditions. In order to benchmark GP regression as a model of microbial population growth, GP prediction error was compared to those from four primary growth models: Gompertz (Zwietering et al. 1990), population logistic regression (Zwietering et al. 1990), Schnute (1981), and Richards (1959; see Methods). All of these primary growth models depend on parameters $\lambda$ and $\mu_{\max }$, corresponding to lag time and maximum growth rate, respectively (Zwietering et al. 1990; Baranyi and Roberts 1995), of a sigmoidal growth curve. The Gompertz, logistic regression, and Richards models also include a parameter for carrying capacity $(A)$. Both the Richards and Schnute models include parameters that modify the sigmoidal shape of the growth curve but do not have direct biological interpretations (Zwietering et al. 1990). The computational time to estimate classical growth parameters was somewhat smaller for primary growth models than for GP regression, but the difference in time is negligible to the researcher (Supplemental Fig. S3).

To test model accuracy of GP regression against primary growth models, data were randomly split into training and test sets including $80 \%$ and $20 \%$ of the data, respectively. We calculated mean squared error (MSE) between test data and model prediction given training data for each model under both standard conditions and oxidative stress. The fit to the data from all models was qualitatively (Fig. 1A) and quantitatively (Fig. 1B) accurate under standard conditions. However, chronic oxidative stress modified the growth trajectory of $H$. salinarum populations such that the data deviated from primary model assumptions (Fig. 1C), and MSE increased by an order of magnitude across all methods besides GP regression (Fig. 1D). GP regression MSE under both standard and stress conditions was significantly lower than MSE for each of the primary models (one-sided $t$-test, $P \leq 10^{-5}$ ) (Fig. 1B,D). Unlike primary models, the difference in MSE between the standard and stress conditions for GP regression was only $2.6 \%$ (one-sided $t$-test, $P=$ 0.90). This shows that B-GREAT models growth data from populations grown under standard and stress conditions with equivalent accuracy.

We next tested the accuracy of GP regression as a function of sampling density, both in the number of observed time points and the number of experimental replicates. We found that GP regression accuracy, measured using MSE, was relatively stable as sampling density decreases, and error did not increase until fewer than 12 replicates or eight time points were used for training (Fig. $1 \mathrm{E}, \mathrm{F})$. The maximum difference in MSE as a function of replicate number was $10.5 \%$, while the maximum error as a function of time points was nearly five times higher with eight time points than with the original 96 time points. The increase in error as a function of a decrease in replicate number was gradual, while the error as a function of time points had a sharp inflection point when fewer than eight time points were used. Generally speaking, these error estimates are useful to guide experimental design for time-series growth data.

\section{GP regression recovers parameters of primary growth models}

To enable a biological interpretation of GP growth curves and a quantitative comparison with primary parametric model output, growth parameters of primary models $-A, \mu_{\max }$, and AUC-were extracted from fitted GP models (see Methods). GP estimates of these parameters under standard growth conditions for the $\Delta$ ura3 parent strain were well correlated with those from Gompertz regression $\left(r^{2}=0.903\right.$ for $\mu_{\max }$ and $r^{2}=0.947$ for $A, P \leq$ $10^{-5}$, Pearson correlation) (Fig. 2A,B). Estimates of $A$ from Gompertz regression were slightly higher than those from GP regression for a subset of samples (Fig. 2B; Supplemental Fig. S4A). Conversely, estimates of $\mu_{\max }$ from GP were higher than those from Gompertz for three growth curves due to instrument noise in the first few time points (Supplemental Fig. S4B). Despite these exceptions, the correlation in parameters was high across models.
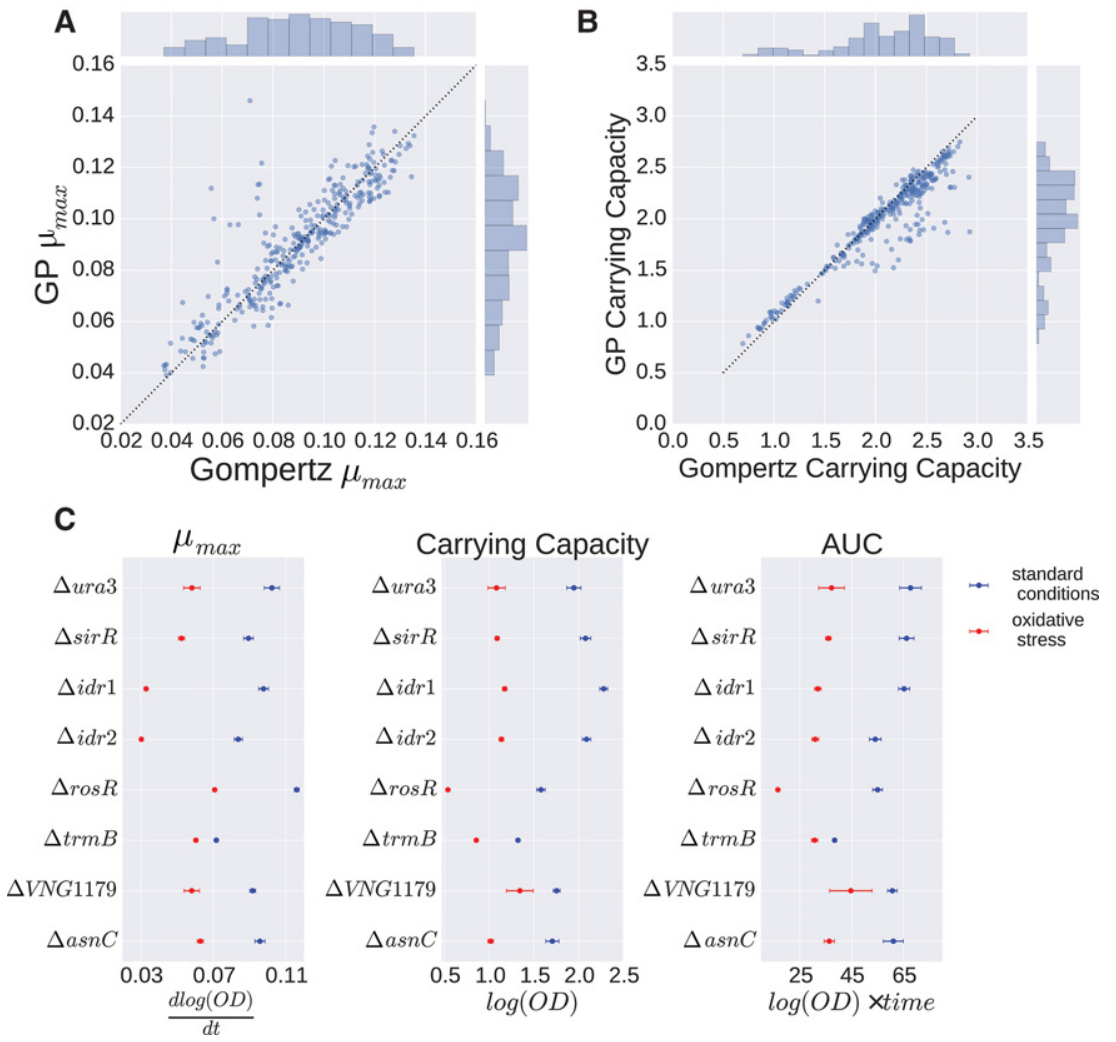

Figure 2. Growth parameters estimated using GP regression. $(A, B)$ Correlation of parameter estimates of $\mu_{\max }(A)$ and carrying capacity $A(B)$ between Gompertz and GP regression. Dotted line represents the line $y=x$. (C) Posterior representations of growth parameters $\mu_{\max }$, carrying capacity, and AUC are shown for each strain under standard conditions (blue) and oxidative stress (red). Points represent posterior mean; error bars, $95 \%$ credible regions. 
GP regression estimates of $A, \mu_{\max }$ and AUC for the $\Delta u r a 3$ parent strain were then compared with parameter estimates from seven TF deletion strains under both standard and oxidative stress conditions. According to these parameters, some mutant strains differed from the $\Delta u r a 3$ parent under standard conditions, while others differed under oxidative stress. For example, $\mu_{\max }$ for the $\Delta t r m B$ strain, a known nutrient responsive regulator, was lower than $\mu_{\max }$ for the $\Delta u r a 3$ strain under standard conditions as expected from previous studies (Schmid et al. 2009; Todor et al. 2013, 2014). Estimates of $A$ and AUC for the $\Delta r o s R$ strain were lower than $A$ and AUC for the other strains. We found significant differences for one or more parameters estimated from the Gompertz model between $\Delta u r a 3$ and TF mutant strains, except for $\triangle a s n C$ under PQ stress ( $t$-test, $P \leq 0.01$; family-wise error rate [FWER] $\leq 0.25$ ) (Supplemental Figs. S5, S6; Supplemental Table S2). Under both standard and oxidative stress conditions, all strains were considered significant for at least one growth parameter (Supplemental Figs. S5, S6). For $A, \Delta$ sirR was the only strain that was not significant under both conditions (Supplemental Figs. S5, S6). These results demonstrate that growth parameters estimated from GP models are biologically relevant and comparable to those estimated using primary models under standard conditions. GP has the added benefit of estimating these parameters accurately for stress conditions, although the biological interpretation may differ from parameters estimated for standard conditions.

\section{B-GREAT identifies known and novel differential growth phenotypes under standard conditions}

We next sought to identify differential growth phenotypes of TF mutants versus the $\Delta u r a 3$ parent strain under standard conditions. Testing for differences in growth phenotypes across strains using classical growth model parameters was difficult: (1) A separate test was conducted for each parameter; (2) comparing variation between multiple parameters was not straightforward because of differences in magnitude (Fig. 2C); and (3) $t$-tests of classical growth parameters were overly sensitive, calling nearly all strains significant for multiple parameters across conditions (Supplemental Figs. S5, S6). To overcome these limitations, we developed a statistical test using Bayes factors (BFs) based on our GP regression model. B-GREAT was designed to capture differences across the entire time series, irrespective of the magnitude and shape of the deviation. Specifically, B-GREAT compares the data likelihood under two models, the null and alternative models. For the sake of efficiency, point estimates of the GP regression hyperparameters are computed instead of integrating over their uncertainty, making our BF estimates approximate (Kass and Raftery 1995; Stephens and Balding 2009). For the null model, $H_{0}$, we used $f$ (time), which indicates that the population growth under the condition of interest is the same between parent and mutant strain. For the alternative model, $H_{A}$, we used OD(time, strain) $=f$ (time, strain), which represents the function of the OD at a given time and for a specific strain, where a strain value of zero or one indicates parent strain or mutant strain, respectively. The covariate strain was added to the model by extending the RBF kernel of the GP to an additional input dimension (Rasmussen and Williams 2006). The alternative model assumes that a given mutant population has a different growth response phenotype than the parent strain while sharing some characteristic shape through the time covariate (Fig. 3A). Typically, larger BFs indicate evidence for the alternative hypothesis, suggesting differential growth across the covariate (Kass and Raftery 1995).
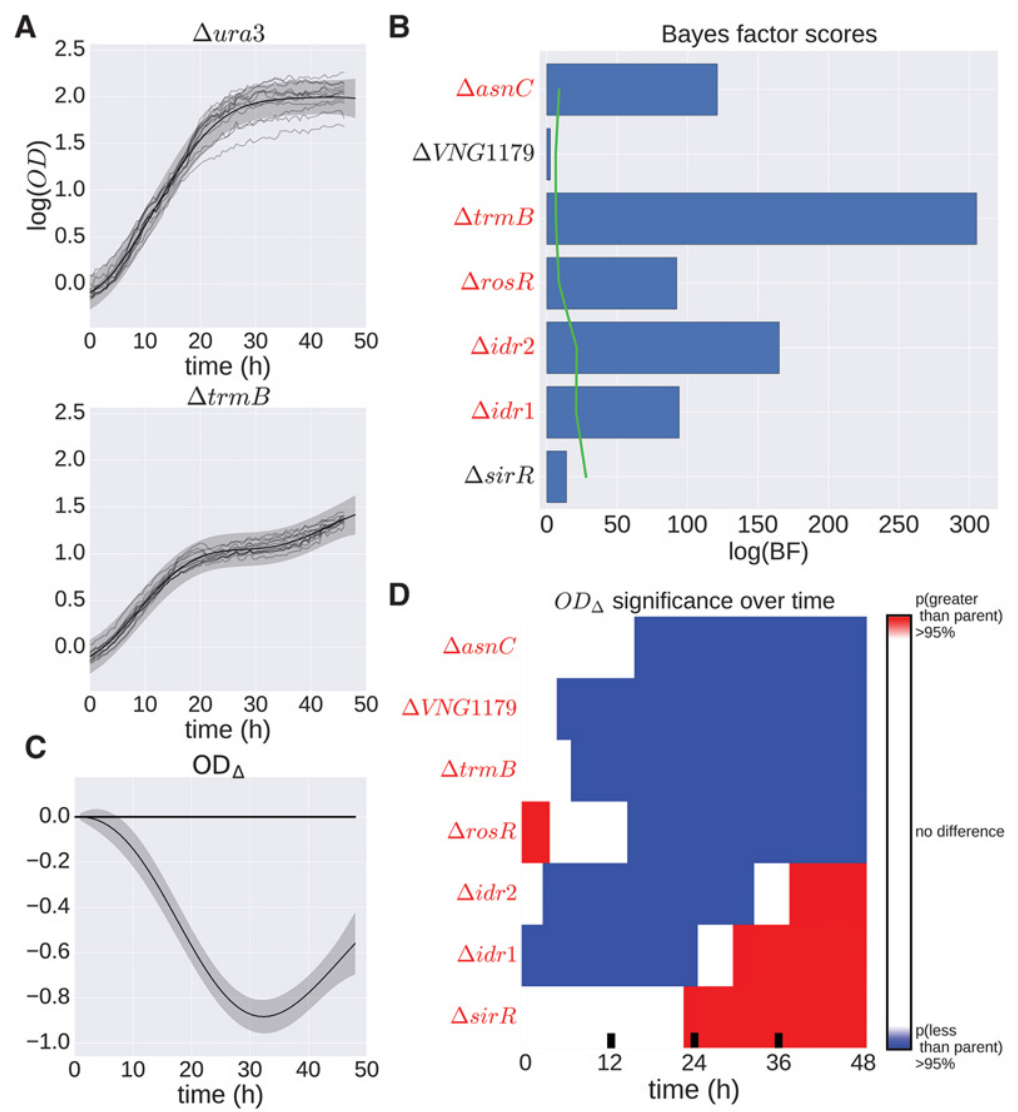

Figure 3. $H$. salinarum mutants with significant growth phenotypes under standard conditions. $(A)$ Population growth data and GP model fit of $H$. salinarum parent strain $\Delta$ ura 3 (top) and $\Delta$ trmB (bottom) under standard growth. Light gray curves represent growth samples of each strain in different wells. Solid black lines and shaded gray regions indicate mean and 95\% credible region of the GP model fit to the growth data, respectively. A single GP model was fit (equation 16) and separate growth predictions made for $\Delta u r a 3$ and $\Delta$ trmB (see Methods). (B) Bayes factors (BFs) for each mutant strain are shown as blue bars. Permuted BF scores representing an FDR $\leq 20 \%$ is indicated by the green line. Strains with a BF score with FDR $\leq 20 \%$ are in red italics. (C) The difference in growth level between $\Delta$ trm $B$ and $\Delta u r a 3$ using the prediction of growth from the GP model. The solid line indicates mean difference, and the shaded region is the $95 \%$ credible region. Regions where the $95 \%$ credible region does not include zero suggest that the growth between the two strains is different at that time point with high probability. $(D)$ Predicted difference between mutant and parent strain population growth using posterior function distributions as in the previous panel. Red and blue regions indicate a $>95 \%$ probability that the mutant population growth is either higher or lower than the parent strain, respectively. Strains with $\mathrm{OD}_{\Delta} 95 \%$ credible region not including zero at any time point are in red italics.

\section{Genome Research}

www.genome.org 
In order to compute the statistical significance for our test of differential growth, we used permutations to calibrate the false-discovery rate (FDR) of our BFs (Stephens and Balding 2009). To do this, we developed a permutation framework to quantify the distribution of the test statistic under a null hypothesis (see Methods). Specifically, across growth data for both parent and mutant strains, the label of strain background was randomly permuted for each time point. Values were permuted so as to maintain the underlying distribution of strain labels present in the original data. We performed 100 permutations that represent an empirical null distribution for each BF test, and BF scores corresponding to FDR $\leq 20 \%$ were considered significant (Stephens and Balding 2009). By using an estimate of the distribution of the test statistic under the null hypothesis, we quantified the FDR for a given test statistic threshold (Mangravite et al. 2013). We performed calibration via permutation in lieu of using a test statistic that has an approximate $\chi^{2}$ distribution for more precise calibration at the cost of additional computation (Fusi and Listgarten 2016).

BF scores calculated from B-GREAT fits on growth curves for each mutant strain represent the overall effect of the strain background on population growth. B-GREAT found that five of the seven TF mutants had significant BFs under standard growth conditions, meaning that the mutant strain showed differential growth compared with the parent strain (FDR $\leq 20 \%$ ), including $\Delta a s n C, \Delta t r m B, \Delta r o s R, \Delta i d r 2$, and $\Delta i d r 1$ (Fig. 3B). To gain further biological insight into the phenotypes of the five strains with differential growth, we developed a second metric, $\mathrm{OD}_{\Delta}$, that quantifies the difference in parent and mutant strain population growth at each time point (see Methods, equation 20) (Supplemental Fig. S7; Benavoli and Mangili 2015). This difference is computed using the posterior estimates of parent and mutant strain growth of the fitted B-GREAT model. As we are interested in differences in the actual growth of strain populations and not in differences arising from noise in growth measurements, $\mathrm{OD}_{\Delta}$ is computed using posterior estimates of the underlying growth function without local Gaussian noise. Specifically, we computed the probability of the mutant strain growth conditioning on the parent strain growth at each observation time point according to the MVN distribution. We thresholded this probability at 95\% to quantify a growth difference between parent and mutant strain at each time point.

As in previous work (Schmid et al. 2009), $\mathrm{OD}_{\Delta}$ showed that $\Delta$ trmB grows more slowly than the $\Delta u r a 3$ parent strain throughout the time course (Fig. 3C,D). In contrast, $\Delta i d r 1$ and $\Delta i d r 2$ grow more slowly than the parent strain during exponential phase but reach higher cell densities during the latter portion of the growth curve (Fig. 3D). $\Delta$ rosR exhibits the opposite growth pattern. The fifth strain with a novel differential growth phenotype, $\Delta a s n C$, is impaired for growth throughout the time course. Although the growth of $\Delta i d r 1, \Delta i d r 2, \Delta r o s R$, and $\triangle a s n C$ strains has been studied during log phase under standard growth conditions previously (Schmid et al. 2011; Sharma et al. 2012; Plaisier et al. 2014), these represent novel stationary phase phenotypes. Taken together, these results demonstrate that B-GREAT and the $\mathrm{OD}_{\Delta}$ metric provide a simple, biologically interpretable test of significance of differential growth that captures the complexity of growth phenotypes.

\section{Identification of differential growth phenotypes in response to oxidative stress}

We next used B-GREAT to quantify the change in population growth of the TF mutants and $\Delta u r a 3$ under chronic oxidative stress. The previous model of growth, $f$ (time, strain), was extended to include an effect of $P Q$ and an interaction term between strain and PQ stress: $f$ (time, strain, $\mathrm{mM} P Q,(\mathrm{mM} P Q \times$ strain)) (equation 17). Here, $\mathrm{mM} P Q \in\{0,1\}$ represents the presence or absence of oxidative stress in the culture (Fig. 4A,B, green curves). The interaction term $\mathrm{mM} P Q \times$ strain $\in\{0,1\}$ is equal to one only for the mutant strain under oxidative stress, and to zero otherwise, and was included to test for differential growth of each mutant strain specific to oxidative stress (Fig. 4B, purple and green curves). The $\mathrm{BF}$ for this condition calculates the relative likelihood of the data with or without the interaction term $\mathrm{mM} P Q \times$ strain (alternative and null models, respectively). This test statistic quantifies differential strain growth under oxidative stress while controlling for differences in growth between parent and mutant strain under standard conditions. The $\mathrm{OD}_{\Delta}$ test was computed as the difference between mutant strain growth with or without the interaction term $\mathrm{mM} P Q \times$ strain (Fig. 4C).

This extended B-GREAT framework detected significantly reduced growth relative to the parent strain for $\Delta$ sirR during the later stages of the time series under oxidative stress $\left(\mathrm{OD}_{\Delta} 95 \% \mathrm{CI}\right.$; BF FDR $\leq 20 \%$ ) (Fig. 4C-E). $\Delta$ sirR was previously implicated in regulating genes involved in metal ion uptake (Kaur et al. 2006), but not in oxidative stress. No other strains were determined to have a significant growth impairment or improvement under PQ stress when differences in strain growth under standard conditions were controlled for in the model (Supplemental Fig. S8). These results indicate that it is straightforward to extend B-GREAT to control for known differential conditions to enable the discovery of novel differential growth phenotypes for previously characterized TF mutant strains.

\section{Meta-analysis improves differential growth phenotype detection}

The strain $\triangle$ ros $R$ is a known oxidative stress regulator that has previously been shown to regulate oxidative stress under both PQ and hydrogen peroxide exposure (Sharma et al. 2012; Tonner et al. 2015). Surprisingly, this strain did not exhibit a significant differential growth phenotype versus the parent strain under oxidative stress in our study (Fig. 4D,E). In order to determine the source of this discrepancy, we compared the growth data for $\Delta$ ros $R$ generated for this study to data from a previous study (Supplemental Fig. S9; Sharma et al. 2012). We observed that $\Delta u r a 3$ reached a higher cell density in stationary phase than $\triangle$ ros $R$ under standard conditions, showing a significant BF score (FDR $\leq 20 \%$ ) in our study (Fig. 3B). Thus, controlling for the differential growth of the strain under standard conditions removed the differential stress condition phenotype. This difference during stationary phase under standard conditions was observed but not quantified in the previous study because only log phase was considered (Sharma et al. 2012).

To combine data from this study and from the previous study, we built a hierarchical GP model of growth that corrects for differences arising between batches of experiments (see Methods) (Hensman et al. 2013). Under this model, a shared growth function $g(\cdot)$ is estimated using a GP whose covariates match those in equation 17 . Then systematic variation between the two studies was modeled as two GPs $f_{1}$ and $f_{2}$, whose means are given by the shared growth function $g(\cdot)$. Under this design, $g$ represents the true growth phenotype of $\Delta$ ros $R$ when corrected for study effects, and $f_{1}$ and $f_{2}$ represent the growth phenotype with study-specific effects included (Fig. 5A,B). From this model, we calculated the difference in $\Delta$ rosR growth with and without the $(\mathrm{mM} P Q \times$ strain) interaction term. Once the variation between studies was corrected for, $\mathrm{OD}_{\Delta}$ indicates that $\triangle$ ros $R$ has a significant growth defect under 

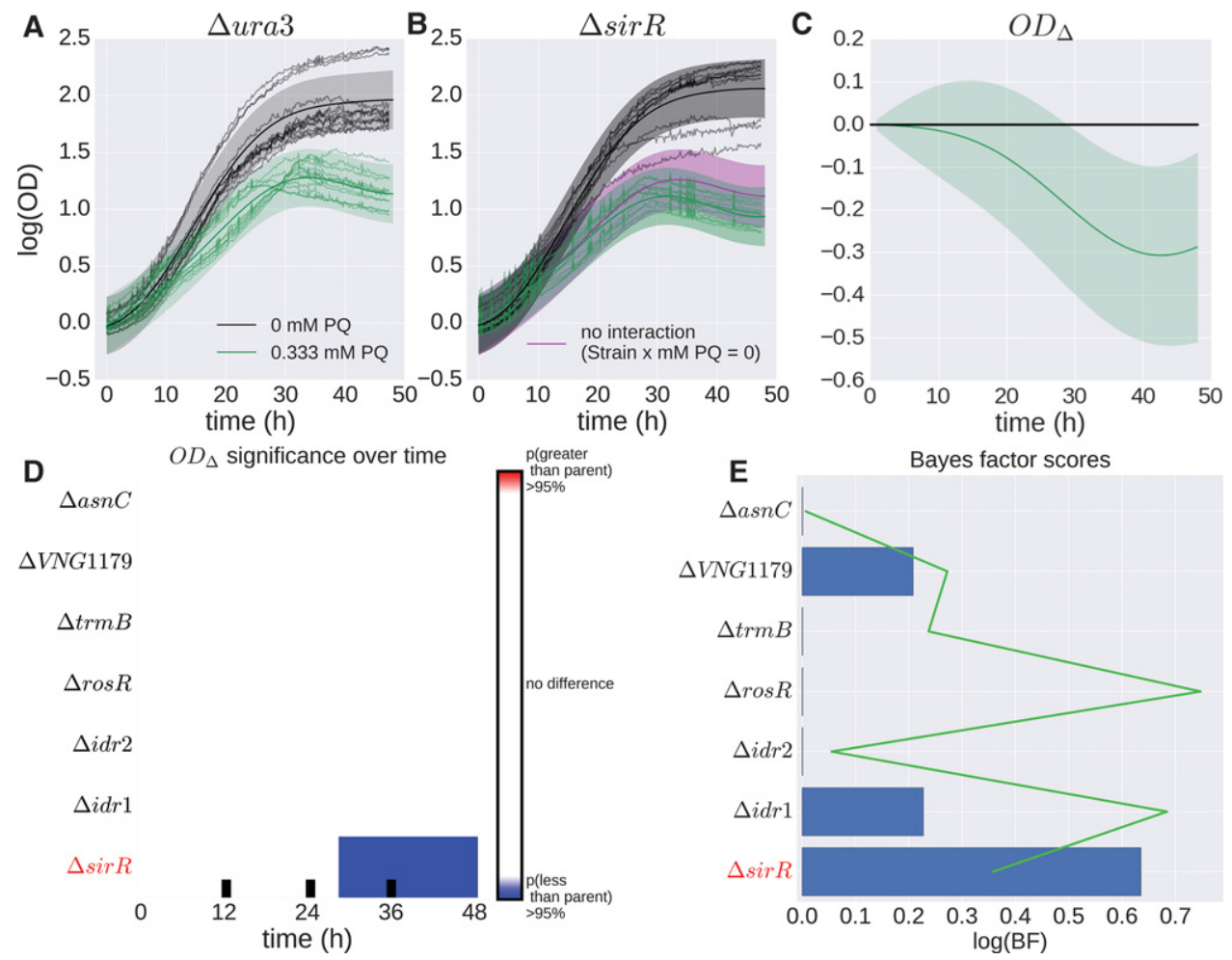

Figure 4. $H$. salinarum mutants with significant growth phenotypes under oxidative stress. $(A, B)$ Example of population growth data from $H$. salinarum for mutant strain $\Delta u r a 3(A)$ and $\Delta \operatorname{sir} R(B)$ under standard conditions (black) and chronic oxidative stress (green). Each curve represents a different sample of an experimental condition. Gaussian process predictions for these conditions are shown as a solid line (mean) and shaded region (variance). The purple line represents the growth prediction when the Strain $\times \mathrm{mM} \mathrm{PQ}$ interaction term is zero. (C) Difference computed between the mutant growth level with interaction term (Strain $\times \mathrm{mM} \mathrm{PQ}=1$ ) and mutant growth without interaction (Strain $\times \mathrm{mM} P Q=0$ ); solid lines represent mean, and shaded regions indicate $95 \%$ credible regions. (D) Functional difference and permuted BF scores for mutant strains in response to oxidative stress. Functional difference is computed between mutant strain with and without an interaction term between mutant and stress condition. $(E) B F$ score and permuted BFs for each strain are shown, where blue bars and green line represent observed BF and FDR $\leq 20 \%$ threshold, respectively. Strains with FDR $\leq 20 \%$ are in red italics.

oxidative stress (Fig. 5C), and the significance of this defect is confirmed with the BF score (FDR $\leq 20 \%$ ) (Fig. 5D). This differential phenotype is consistent with the known function of the RosR TF as a genome-wide regulator of gene expression in response to oxidative stress (Tonner et al. 2015). These results demonstrate that this hierarchical model effectively combines cross-study data and corrects for study-specific effects, recapitulating the known phenotype of $\Delta$ ros $R$ under PQ stress (Fig. 5C,D).

\section{B-GREAT identifies significant growth phenotypes across strains of yeast}

To test the efficacy of B-GREAT as a general microbial population growth model, we applied our method to a large compendium of yeast growth profiles in which 96 domesticated and wild strains of Saccharomyces cerevisiae and Saccharomyces paradoxus were grown in various stress conditions (Liti et al. 2009). We used BGREAT to test for differential growth between the control strain, BY4741, and all other strains under PQ stress and cycloheximide stress (Supplemental Figs. S10 and S11, respectively). Under both conditions, strains identified as having significant differential growth (FDR $\leq 20 \%$ ) by B-GREAT were significantly overrepresented by $S$. paradoxus strains relative to $S$. cerevisiae $(P \leq 0.05$, hypergeometric test). While $S$. paradoxus strains make up a minority of the strains in the data (37.5\%), they constituted the majority of the strains with differential growth in response to PQ $(65 \%, P \leq$ $\left.2.3 \times 10^{-7}\right)$ and cycloheximide $\left(79.3 \%, P \leq 1.8 \times 10^{-9}\right)$ (Fig. 6 ; Supplemental Figs. S10, S11). The increased resistance of $S$. paradoxus strains to cycloheximide relative to that of $S$. cerevisiae strains had been previously detected (Liti et al. 2009). However, in our BGREAT analysis, we also detected significantly decreased resistance of $S$. paradoxus to $\mathrm{PQ}$, a novel finding.

The yeast strain with the highest BF score, S. paradoxus G4650, showed severely inhibited growth in response to PQ stress compared with the BY4741 control (Fig. 6A-C). G4650 was isolated from fossilized guano in Italy, with no previously reported sensitivity to oxidative stress (Liti et al. 2009). When we extended the analysis to all strains in the data set, we saw a trend for $S$. paradoxus strains to grow poorly under PQ exposure compared with the BY4741 control (Fig. 6D). Together, these results show that BGREAT recapitulates known biology and identifies new differential phenotypes from previous studies on large collections of strains with diverse genetic backgrounds.

\section{Discussion}

In this study, we developed B-GREAT, a general model of microbial population growth using GP regression. B-GREAT overcomes the limitations of primary parametric models and enables discovery of novel growth phenotypes for genetically and

\section{Genome Research}

www.genome.org 
A
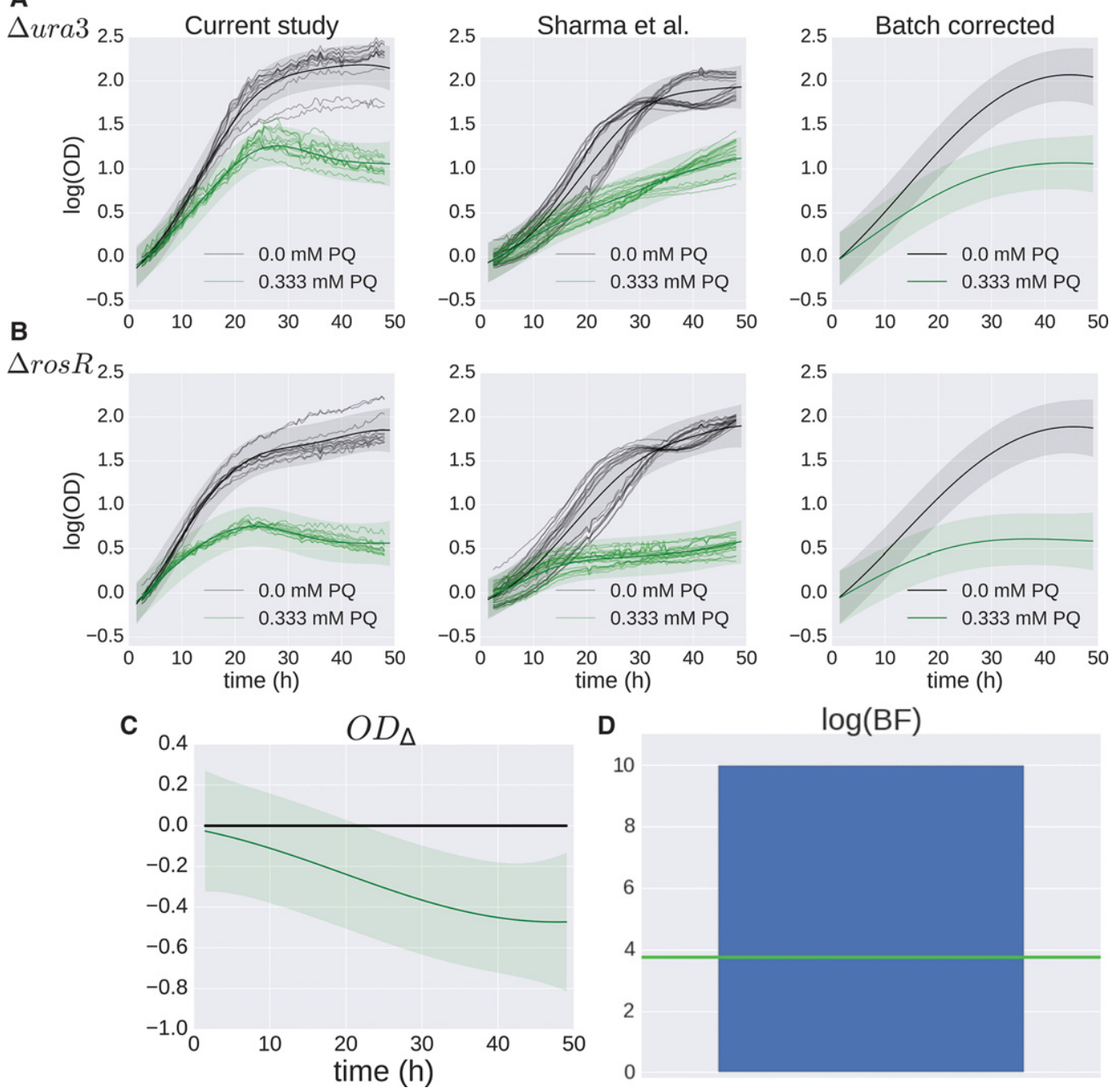

Figure 5. B-GREAT model of $\Delta \operatorname{ros} R$ growth in response to oxidative stress across multiple studies. $(A, B) \Delta u r a 3(A)$ and $\Delta \operatorname{ros} R(B)$ growth data under standard conditions (black) and oxidative stress (green). Individual samples from this study (left) and previously published data (center) (Sharma et al. 2012) are shown as shaded lines. The B-GREAT model prediction for each condition is shown as solid line and shaded region for mean and $95 \%$ credible region, respectively. The growth prediction for the underlying growth function estimated across studies is shown in the right column. (C) The difference between $\Delta \operatorname{ros} R$ and $\Delta u r a 3$ growth for the underlying growth function corrected for batch effects, which shows an increased susceptibility of $\Delta$ ros $R$ to oxidative stress relative to the parent strain. $(D) \log (\mathrm{BF})$ compared with permuted scores from the null distribution. Blue bar and green line represent observed $\mathrm{BF}$ and $\mathrm{FDR} \leq 20 \%$ threshold, respectively.

environmentally perturbed microbial populations. We showed that B-GREAT is equivalently accurate under nonstandard conditions (Fig. 1B); moreover, its model accuracy is resilient to decreases in observation sampling (Fig. 1E,F). GP regression can recover growth statistics of log phase $\left(\mu_{\max }\right)$ and stationary phase (carrying capacity, A), enabling direct comparison of these variables to results from primary growth models (Fig. 2). Our comparisons demonstrated that GP regression outperforms primary parametric growth models in capturing growth, both under standard conditions and under nonstandard stress conditions (Fig. 1).

In our results, we highlighted important properties of the BGREAT method for modeling microbial growth. GP models allow the inference of smooth underlying growth functions through the length-scale parameter, explicitly accounting for experimental noise. This is in contrast to other recent models such as linear spline fitting in generalized additive models (GAMs), which are sensitive to technical variation (Sekse et al. 2012), or polynomial splines in the grofit package, which require cross-validation to estimate parameters (Kahm et al. 2010). Given the large proportion of cellular machinery whose production correlates linearly with growth rate (Pedersen et al. 1978; You et al. 2013), differentiating general growth impairments from specific, stress-related impairments is important for biological interpretation of model fits. GP regression enables this process by easily incorporating multiple dimensions through the addition of length-scale parameters for each covariate, modeling and controlling for an arbitrary number of covariates (Fig. 4). For example, the number of strains with differential effects increases from one to five out of seven if the $\mathrm{mM}$ $\mathrm{PQ} \times$ strain interaction term is removed (Fig. 4D; Supplemental Fig. S12), demonstrating the importance of this covariate. Additionally, GP regression may be extended to model data in which experimental variance is more complicated than simple independent Gaussian noise (Fig. 5; Shah et al. 2014). 

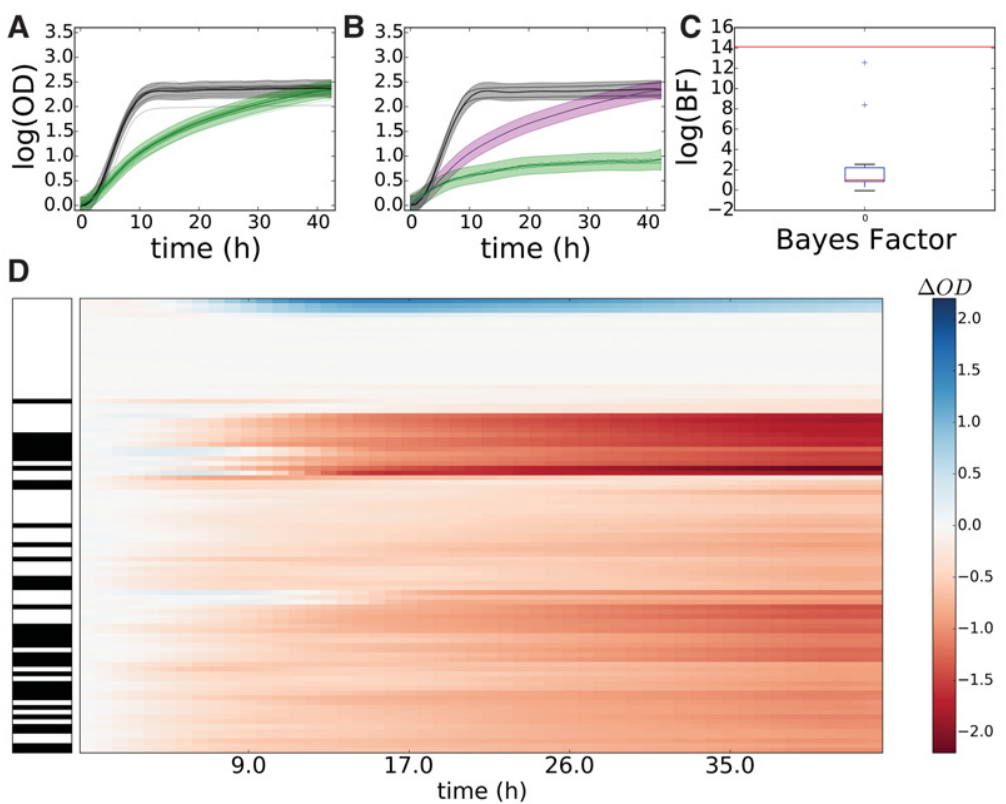

Figure 6. B-GREAT identifies significant growth phenotypes in yeast strains in response to paraquat. $(A$, $B$ ) Control strain BY4741 (A) and Saccharomyces paradoxus strain G4650 (B) growth under standard conditions (black) and under paraquat stress (green). Solid lines represent experimental data, and shaded regions represent B-GREAT model predictions. Purple-shaded region represents $95 \%$ credible region of B-GREAT prediction of G4650 in the absence of stress interaction (strain $\times$ stress $=0$ ). (C) log(BF) (red line) and permuted log(BF)s (boxplot) of G4650 under paraquat stress according to B-GREAT. $(D) \mathrm{OD}_{\Delta}$ scores of all yeast strains under paraquat exposure. Left column corresponds to $S$. cerevisiae (white) or S. paradoxus (black) strains. Center column represents magnitude of calculated $\mathrm{OD}_{\Delta}$ over time for each strain.

We showed in our results in $H$. salinarum that the B-GREAT $\mathrm{BFs}$ and $\mathrm{OD}_{\Delta}$ may be used in combination to characterize differences in growth. In particular, BFs provide an overall metric of growth phenotype significance, and $\mathrm{OD}_{\Delta}$ quantifies the difference between parent and mutant strain growth across the time course. In general, we find that the use of BFs is a conservative method of finding significantly different growth phenotypes relative to $\mathrm{OD}_{\Delta}$. For example, there is one case in which $\mathrm{OD}_{\Delta}$ is significant but the BF is not (e.g., $\Delta V N G 1179 C$ ) (Fig. 3). As such, $\mathrm{OD}_{\Delta}$ and $\mathrm{BF}$ tests provide two tiers of statistical confidence, providing a stringent test to detect phenotypic differences while correcting for variability in the data. By using $\mathrm{BF}$ and $\mathrm{OD}_{\Delta}$ together, an experimental researcher can prioritize strains or conditions to pursue for further study.

B-GREAT recapitulates known biology and discovers previously uncharacterized phenotypes. We confirmed the known growth defect for $\triangle$ trmB under standard conditions (Fig. 3B-D), which results from its function as a master regulator of metabolic pathways (Schmid et al. 2009; Todor et al. 2013, 2014, 2015). In contrast, the $\triangle a s n C$ oxidative stress phenotype observed previously (Plaisier et al. 2014) was not recapitulated here, likely because the growth defect of this mutant under standard conditions explains the difference in growth during stress (Fig. 3C; Supplemental Fig. S8), which was not corrected for in the previous study (Plaisier et al. 2014). Finally, we identified a previously undiscovered relationship between $\Delta s i r R$ and oxidative damage (Fig. 4). SirR regulates metal uptake transporters at the level of transcription (Kaur et al. 2006), repressing manganese uptake transporters under replete conditions. This regulatory link between metal homeostasis and oxidative stress is well-established in bacterial and eukaryotic organisms (Imlay 2003) but is only beginning to be appreciated in archaeal species (Zhu et al. 2013).

B-GREAT was next applied to population growth data of diverse yeast species under different stress conditions, identifying a previously uncharacterized difference between the growth of $S$. cerevisiae and $S$. paradoxus in response to PQ exposure (Supplemental Fig. S10). Our results also recapitulate the known resistance of $S$. paradoxus to cyclohexamide (Supplemental Fig. S13; Liti et al. 2009). S. paradoxus harbors higher levels of ROS (Deregowska et al. 2015), and our results suggest that this may lead to a higher susceptibility to oxidative stress. Many other large-scale population growth studies have been performed to differentiate biological function through population growth phenotypes in the yeast community, and we anticipate that future applications of B-GREAT will highlight additional results from these studies (Warringer et al. 2003; Fernandez-Ricaud et al. 2005).

In future work, B-GREAT will be applied to many problems in testing functional data for significant differential responses to perturbation. Gene expression time-series studies could benefit from this method, where each gene can be tested for differential dynamic profiles between conditions of interest (Bar-Joseph et al. 2012). Population genome-wide association studies (GWAS) are also a potential application of this method to detect the effect of different loci on function responses (Fusi and Listgarten 2016). By adding new covariates, B-GREAT may also be extended to model continuous effects such as dose response (Sekse et al. 2012; Di Veroli et al. 2015; Twarog et al. 2016). By using a time-dependent variance parameter rather than a stationary kernel, B-GREAT may also be extended to model functional data in which heterogeneity between samples is a function of time (Cao et al. 2010). B-GREAT provides a strong foundation to perform and extend the interpretable analysis of the large and growing quantity of dynamic, functional biological data.

\section{Methods}

\section{H. salinarum growth data}

Growth of seven TF mutant strains for H. salinarum, each deleted in-frame for a TF-encoding gene, and the isogenic $\Delta u r a 3$ parent strain was measured (Table 1). Details regarding construction of these mutants were described in prior work (Kaur et al. 2006; Schmid et al. 2009, 2011; Sharma et al. 2012; Plaisier et al. 2014). Cultures were inoculated into complete medium (CM; $250 \mathrm{NaCl}, 20 \mathrm{~g} / \mathrm{L} \mathrm{MgSO}_{4} \cdot 7 \mathrm{H}_{2} \mathrm{O}, 3 \mathrm{~g} / \mathrm{L}$ sodium citrate, $2 \mathrm{~g} / \mathrm{L} \mathrm{KCl}$, $10 \mathrm{~g} / \mathrm{L}$ peptone), grown to stationary phase, and then diluted to $\mathrm{OD} \sim 0.05$ for growth analysis. OD at $600 \mathrm{~nm}$ of 200 independent cultures was measured every $30 \mathrm{~min}$ for $48 \mathrm{~h}$ using a Bioscreen C (Growth Curves USA). Growth of each strain under each experimental condition was measured in at least biological quadruplicate (from independent colonies) and technical triplicate

\section{Genome Research}

www.genome.org 
(independent cultures from the same colony), for a total of 12 replicates. Standard and chronic oxidative stress conditions were tested for all mutants. Standard conditions were defined as $42^{\circ} \mathrm{C}$ with 225 r.p.m. shaking under ambient light in rich CM medium (Yao and Facciotti 2011). Chronic oxidative stress was induced with $0.333 \mathrm{mM} \mathrm{PQ}$, a redox cycling drug that permeates the cell membrane, that was added at the inoculation of the Bioscreen experiment.

Prior to statistical analysis, OD data were $\log _{2}$ transformed and scaled by the estimate of starting OD as follows. Data from growth experiments were grouped by their strain and media composition (e.g., $\Delta u r a 3$, standard growth). This corresponds to the 12 replicates comprising four biological replicates and three technical replicates. Then OD measurements from the first 10 time points within each group were fit with a polynomial regression of degree five. The OD value at time $=0$, as estimated by the polynomial regression, was then subtracted from all data points in the group in order to normalize the starting growth levels at zero for all conditions.

\section{H. salinarum data as input to B-GREAT}

Input to the GP model corresponds to measurements $Y_{t, c, r}$ for a given time $(1 \leq t \leq T)$, condition $(1 \leq c \leq C)$, and replicate $(1 \leq r \leq R)$. For standard conditions, time points were taken at 4 -h increments across a 48-h experiment. This resulted in 12 observations from each replicate. Additionally, growth measurements from both parent strain and each mutant strain were included $(C=2)$. A total of $T \times R \times C=288$ observations was used for training each GP model under standard conditions. For oxidative stress, time points were taken every $6 \mathrm{~h}$, for a total of eight time points for each replicate. The decrease in time samples used in the oxidative stress models was necessary to incorporate the increase in conditions for both standard and oxidative stress growth. Specifically, conditions include growth for both parent and mutant strain under both standard and oxidative stress conditions $(C=4)$. This corresponds to a total of 384 observations for each GP model under oxidative stress.

\section{Yeast population growth data}

Population growth data for 96 yeast strains were collected from a previous study (Liti et al. 2009). One hundred eighty-six conditions are represented in the data set covering various nutrient and stress conditions. For each condition, a minimum of eight replicates for the control strain, BY4741, were available and two replicates of each yeast strain. Measurements were taken every 20 min for $48 \mathrm{~h}$, leading to 144 time points per replicate.

\section{GP regression of microbial population growth data}

GP regression is a probability distribution on arbitrary functions mapping $x$ to $f(x)$ (Rasmussen and Williams 2006). When observations of $f(x)$ are distorted with IID Gaussian noise, multiple observations of the function are distributed as a multivariate Gaussian

$$
y(x) \sim N(\mu(x), \Sigma) .
$$

In our application, $x$ represents time and $y(x)=\log \mathrm{OD}(x)$ represents the log-transformed OD measurement at time $t$. A GP model requires specification of a mean function $\mu(x)$ and kernel function $\Sigma_{i, j}=\kappa\left(x_{i}, x_{j}\right)$, which defines the positive definite covariance matrix $\Sigma$. In this work, the mean function was set to zero across inputs, $\mu(x)=0$, as is standard (Rasmussen and Williams 2006). For the kernel, we used a RBF with time point-specific independent
Gaussian noise:

$$
\kappa\left(x_{i}, x_{j}\right)=\sigma_{\mathrm{RBF}}^{2} \cdot \exp \left(\frac{-\left\|x_{i}-x_{j}\right\|^{2}}{\ell^{2}}\right)+\sigma_{\text {nugget }}^{2} \cdot \delta_{x_{i}=x_{j}} .
$$

Here, $x_{i}$ and $x_{j}$ are two time points; $\sigma_{\mathrm{RBF}}^{2}$ is the RBF variance parameter; $\sigma_{\text {nugget }}^{2}$ is the Gaussian variance at a single time point $t$ (called the nugget); $\delta_{x_{i}=x_{j}}$ is an indicator function, which is equal to one when $x_{i}=x_{j}$ and to zero otherwise; and $\ell$ is the RBF length scale parameter, which dictates the smoothness of the function $f(x)$ through the GP distribution. Kernel function parameters $\theta=\left\{\sigma_{\mathrm{RBF}}^{2}, \sigma_{\text {nugget }}^{2}, \ell\right\}$ were optimized by maximizing the likelihood of the data marginalized over the latent function $f(x)$ with respect to each parameter (Rasmussen and Williams 2006). All GP regression models were built and optimized using the GPy package (version 0.8.8) for Python (http://github.com/SheffieldML/GPy).

\section{Other kernels tested}

Two other kernels were tested for comparison to RBF kernels, the Matérn and linear kernels. Matérn kernels are defined as

$$
k(r)=\sigma^{2}(1+\sqrt{3} r) \exp (-\sqrt{3} r) \quad \text { where } r=\sqrt{\frac{\left(x_{i}-x_{j}\right)^{2}}{\ell^{2}}} .
$$

Linear kernels are defined as

$$
k\left(x_{i}, x_{j}\right)=\sigma_{i}^{2} x_{i} x_{j}
$$

Model fit for each kernel was assessed with the data likelihood of the optimized GP model and also using the BIC (Neath and Cavanaugh 2012). BIC is calculated as

$$
-2 \times \log (L)+k \times \log (n)
$$

where $L$ is the likelihood of the data, $k$ is the number of hyperparameters for each kernel, and $n$ is the number of data points.

\section{GP growth curve metrics}

The growth curve metrics $\mu_{\max }$ and carrying capacity $A$ were calculated from the maximum a posteriori (MAP) estimates of either log $(\mathrm{OD})$ or $(d / d x) \log (\mathrm{OD})$ for carrying capacity and $\mu_{\max }$, respectively. MAP estimates of $\log (\mathrm{OD})$ are given by the model in equation 1 , by taking the MAP growth level using the fitted model. In order to calculate a MAP estimate of $(d / d x) \log (\mathrm{OD})$, we estimate $(d / d x) \log (\mathrm{OD})$ using GP regression. The RBF kernel is infinitely differentiable, so derivative observations of a GP regression model are also distributed as a GP as follows (Solak et al. 2003):

$$
\frac{d}{d x} \log (\mathrm{OD}) \sim \mathrm{GP}\left(\frac{d}{d x} \mu, \frac{d}{d x} \Sigma\right),
$$

where

$$
\frac{d}{d x} \mu=0
$$

and

$$
\begin{aligned}
\frac{d}{d x} \kappa\left(x_{i}, x_{j}\right)= & \frac{2 \cdot \sigma_{\mathrm{RBF}}^{2}}{\ell} \times\left(1-\frac{2 \cdot\left(x_{i}-x_{j}\right)^{2}}{\ell}\right) \\
& \cdot \exp \left(\frac{-\left\|x_{i}-x_{j}\right\|^{2}}{\ell}\right) .
\end{aligned}
$$

The GP model of $(d / d x) \log (\mathrm{OD})$ was used to calculate the MAP estimate of $(d / d x) \log (\mathrm{OD})$ as an estimate of $\mu_{\max }$.

The estimate of AUC was calculated as a metric of the $\log (\mathrm{OD})$ distribution as a function of time $t$. The posterior distribution of 
OD measurements over time is predicted as a MVN, $\log (\mathrm{OD})(t) \sim$ $\left.N(\mu(t)), \Sigma\left(t, t^{\prime}\right)\right)$. Predictions were made at 50 evenly spaced time points during the growth curve, and the linear transformation was made of $\log (\mathrm{OD})(t)$ on the vector $a=\{\Delta t, \Delta t, \ldots\}$, where $\Delta t$ is the space between predicted time points. This linear transformation is then an approximation of the AUC for the condition, with a normal distribution AUC $\sim N\left(a \cdot \mu, a \Sigma a^{T}\right)$ (Todor et al. 2014).

\section{Primary growth models}

We compared the predictions from the fitted GP regression model to predictions from four primary growth curve models: Gompertz, population logistic, Schnute, and Richards regression (Zwietering et al. 1990). All model parameters were optimized with the curve_fit function of the scipy Python package, which estimates function parameters using damped least squares (Millman and Aivazis 2011). Input data were randomly divided into training $(80 \%)$ and test (20\%) sets for each of the 721 total growth curves in the data set. The MSE of each model fit with respect to the $20 \%$ held out test data was calculated as the difference between prediction and test data from models estimated using the training data:

$$
\operatorname{MSE}(y, m)=\frac{1}{T} \sum_{t=1}^{T}\left(y_{t}-m_{t}\right)^{2},
$$

where $y_{t}$ and $m_{t}$ correspond to raw data and model predictions at the $t$ th time point, respectively. Model prediction $m_{t}$ was the posterior mean of the fitted GP, and primary growth model predictions were taken from the growth level predicted by the estimated parameters. By use of a one-sided sample $t$-test, MSE for GP regression fit was compared separately to each of Gompertz, population logistic, Schnute, and Richards regression fits. These primary models were selected to compare against the most widely used primary models in modeling microbial population growth (McKellar and $\mathrm{Lu}$ 2003). Additionally, the models chosen have been shown to be related to one another through specific constraints on parameters. For example, Gompertz regression can be recovered from the Schnute model with parameters $a>0$ and $b=0$ (Zwietering et al. 1990). Therefore, we can observe the improvement of primary model accuracy as we add additional parameters.

\section{Gompertz regression}

$$
y(t)=A \cdot \exp \left[-\exp \left[\frac{\mu_{\max } \cdot e}{A}(\lambda-t)+1\right]\right],
$$

where $A$ is the carrying capacity, $\mu_{\max }$ is the maximum growth rate, and $\lambda$ is lag time (Zwietering et al. 1990).

\section{Population logistic regression}

$$
y(t)=A \cdot\left[1+\exp \left(\frac{4 \cdot \mu_{\max }}{A}(\lambda-t)+2\right)\right]^{-1},
$$

where $A$ is the carrying capacity, $\mu_{\max }$ is the maximum growth rate, and $\lambda$ is lag time. (Zwietering et al. 1990).

$$
\begin{aligned}
& \text { Schnute model } \\
& \qquad y(t)=\mu_{\max } \cdot \frac{1-b}{a} \cdot\left[\frac{1-b \cdot \exp (a \cdot \lambda+1-b-a \cdot t)}{1-b}\right]^{1 / b},
\end{aligned}
$$

where $\mu_{\max }$ is the maximum growth rate, $\lambda$ is lag time, and $a$, and $b$ are parameters that affect the growth curve shape but do not have direct biological interpretation (Zwietering et al. 1990).
Richards model
$y(t)=A \cdot\left[1+v \cdot \exp (1+v) \cdot \exp \left(\frac{\mu_{\max }}{A} \cdot(1+v) \cdot\left(1+\frac{1}{v}\right) \cdot(\lambda-t)\right)\right]^{-1 / v}$,

where $A$ is the carrying capacity, $\mu_{\max }$ is the maximum growth rate, $\lambda$ is lag time, and $v$ is a parameter that affects the growth curve shape but does not have direct biological interpretation (Zwietering et al. 1990).

\section{Testing for significant parameter differences in classical models}

Growth parameters $\mu_{\max }$ and carrying capacity were tested under standard condition and oxidative stress by taking the corresponding parameter estimates for $\Delta u r a 3$ and each mutant strain and computing a $t$-test for significant differences between the two populations of parameter estimates.

\section{Testing for differential growth using BFs}

We developed an approximate BF test statistic to quantify possible differences between a pair of growth conditions $\mathrm{BF}_{\text {strain }}$ (Kass and Raftery 1995; Stephens and Balding 2009). BFs were calculated as the ratio of data likelihoods between an alternative model $\left(H_{a}\right)$ and a null model $\left(H_{0}\right)$ :

$$
\mathrm{BF}=\frac{p\left(Y \mid H_{a}\right)}{p\left(Y \mid H_{0}\right)}
$$

Larger values of the BF indicate a higher relative likelihood under the alternative model and provide evidence for the alternative model representing the data better than the null model.

Specifically, we designed three different BF test statistics to measure differences in population growth across covariates. Under standard conditions, we use $\mathrm{BF}_{\text {strain }}$, in which the null model $H_{0}$ assumes that growth is the same across the parent and mutant strain; the alternative model $H_{a}$ captures growth between the parent and mutant strain separately. A high BF then suggests that the growth phenotype is different across strains. We designed a second test for differential growth in the presence of oxidative stress, $\mathrm{BF}_{\text {stress, }}$, where the alternative model included an interaction term between genetic effect and oxidative stress. High BF scores under this condition indicate that the mutant strain has a differential growth phenotype relative to the parent strain under oxidative stress. We designed a third test for differential growth across two separate studies, $\mathrm{BF}_{\text {study, }}$ which performs the same test as $\mathrm{BF}_{\text {stress }}$ but shares statistical strength across batches of growth measurements using a hierarchical GP model.

A FDR for each BF was calculated using an estimate of the null $\mathrm{BF}$ distribution, representing BF scores when no significant growth effect between the two conditions is observed. For a single growth experiment, $Y=\left\{y_{1}, y_{2}, \ldots y_{T}\right\}$ and corresponding time, genetic background, and other covariates $X=\left\{x_{1}, x_{2}, \ldots x_{T}\right\}$, each $x_{t}=\{$ time, strain, ...\}, were randomly assigned a value for strain that preserved the original distribution of strain values in $X$. One hundred permutations of the data indices following this design were constructed, and a BF score was calculated for each permutation. The distribution of permuted BF scores was used as an estimate of the null distribution of the test statistic, and a BF score that exceeded $80 \%$ of permuted scores (corresponding to FDR $\leq 20 \%$ ) was selected as significant.

More generally, FDR is calculated using permutations, for a given $\mathrm{BF}$ threshold $c$, as follows:

$$
\operatorname{FDR}(c)=\frac{\left|\mathrm{BF}_{\text {perm }}>c\right|}{\left|\mathrm{BF}_{\text {real }}>c\right|}
$$

\section{Genome Research \\ www.genome.org}


which approximates the FDR, i.e., the number of false positives over the total number of discoveries, for threshold $c$. In this case, there is a single $\mathrm{BF}_{\text {real }}$ for 100 permuted BFs, so we multiplied the $\mathrm{BF}_{\text {real }}$ count by 100 for this computation.

\section{Differential mutant growth phenotypes}

The effects of gene deletion on growth were modeled as experimental effects by extending the input variable $x$, originally representing time, to include perturbations as additional covariates in the GP regression model. The RBF kernel function was extended to handle the additional covariates by using an automatic relevance determination (ARD) prior to induce sparsity on the weighted contribution of each of the K covariates (MacKay 1992; Tipping 2001; Rasmussen and Williams 2006; Neal 2012):

$$
K\left(x_{i}, x_{j}\right)=\sigma^{2} \cdot \exp \left(\sum_{k=1}^{K} \frac{\left\|x_{i, k}-x_{j, k}\right\|}{\ell_{k}}\right),
$$

where each $\ell_{k}$ is the length-scale for the $k$ th covariate. These length-scale parameters are then interpretable in terms of quantifying the relative contribution of each of the $k$ covariates. Genetic background was incorporated into the model covariates as a Boolean variable $x_{\text {strain }} \in\{0,1\}$, where a value of zero indicates parent strain and one indicates mutant strain. For standard growth conditions, $x$ has the form

$$
x=\{\text { time, strain }\},
$$

whereas the null model contains no strain information: $x=\{$ time $\}$. The BF then quantified the improvement in data likelihood of the GP regression model, including the strain information versus omitting strain information; when modeling strains separately improved the data likelihood, this indicated that there was differential growth across strains.

\section{Differential response to stress conditions across strains}

Differential growth in response to PQ exposure was tested by extending the covariates to include two additional covariates. The first covariate, $\mathrm{mM} P Q \in\{0,1\}$, represents the presence (1) and absence $(0)$ of PQ stress. The second covariate, $\mathrm{mM} P Q \times$ strain $\in$ $\{0,1\}$ is an interaction term between mutant strain and stress condition, computed by multiplying the strain covariate with the $\mathrm{mM}$ $\mathrm{PQ}$ covariate. $\mathrm{mM} \mathrm{PQ} \times$ strain covariate was one only for growth measurements made under oxidative stress for the mutant strain and was zero otherwise. The test for significant growth phenotypes was then made using models including or excluding the $\mathrm{mM} P Q \times$ strain interaction term. Specifically, the input $x$ for the PQ condition had the form

$$
x=\{\text { time, strain, } \mathrm{mM} P \mathrm{PQ},(\mathrm{mM} \mathrm{PQ} \times \text { strain })\} .
$$

The null model, where there is no interaction between strain and stress condition, corresponds to

$$
x=\{\text { time, strain, } \mathrm{mM} P \mathrm{PQ}\}
$$

\section{Modeling batch effects and testing for differential effects across studies}

Growth data for $\Delta$ ros $R$ under standard conditions and oxidative stress were collected both in this study and in a previous study (Sharma et al. 2012). We modeled the joint growth data from both studies with a hierarchical GP model (Hensman et al. 2013).
Under this model, the underlying growth function is modeled with a GP: $g(x) \sim \mathrm{GP}\left(\mu_{g}, K_{g}\right)$. Different batch observations of this function are drawn from a GP with mean equal to $g(x): f(x) \sim \mathrm{GP}$ $\left(g(x), K_{f}\right)$.

Growth data for $\Delta r o s R$ and the parent strain were modeled by replicate functions $f_{1}$ and $f_{2}$, representing data from our study and the previous study, respectively. The GP models for $f_{1}, f_{2}$, and $g$ all follow the design in equation 17 . BF scores in both cases were calculated as the difference in log likelihood for GP models accounting for strain variation interacting with oxidative stress $\left(H_{A}\right.$; equation 17) and those that do not interact with oxidative stress $\left(H_{0}\right.$; equation 18$)$. The BF permutation was performed as described above.

\section{Computing differences between population growth across time series $\left(O D_{\Delta}\right)$}

The difference between mutant and parent strain functions across time points were defined by the variable $\mathrm{OD}_{\Delta}$. The variable $\mathrm{OD}_{\Delta}$ is the difference in mutant strain growth and parent strain growth at each time point $t_{k}$. $\mathrm{OD}_{\Delta}$ was calculated using the noiseless latent mean function for population growth rather than the noisy observations. In other words, we use the latent function $f: \log$ $(\mathrm{OD})=f(t)+\varepsilon$, where $f(t)$ is the smooth underlying growth function and $\varepsilon$ represents random noise. $\mathrm{OD}_{\Delta}$ at a specific time point $t_{k}$ is then the difference between the growth of the mutant strain $f_{m}$ and the parent strain $f_{p}$ :

$$
f_{m}\left(t_{k}\right)-f_{p}\left(t_{k}\right)
$$

where $f_{m}$ and $f_{p}$ are estimated from the posterior distribution of the trained GP model. Finally, $\mathrm{OD}_{\Delta}$ also corrects for differences between parent and mutant strain at the start of the experiment, $t_{0}$, by subtracting their respective growth levels at that time point:

$$
\mathrm{OD}_{\Delta}\left(t_{k}\right)=\left(f_{m}\left(t_{k}\right)-f_{p}\left(t_{k}\right)\right)-\left(f_{m}\left(t_{0}\right)-f_{p}\left(t_{0}\right)\right)
$$

The four variables needed to calculate $\mathrm{OD}_{\Delta}$-i.e., $f_{k}=\left\{f_{m}\left(t_{k}\right)\right.$ $f_{m}\left(t_{0}\right), f_{p}\left(t_{k}\right)$, and $\left.f_{p}\left(t_{0}\right)\right\}$-are defined by a joint MVN distribution predicted by the fitted GP:

$$
\begin{aligned}
f_{k} & =\left[f_{m}\left(t_{k}\right), f_{m}\left(t_{0}\right), f_{p}\left(t_{k}\right), f_{p}\left(t_{0}\right)\right]^{T} \\
& \sim N\left(\left[\mu_{m}\left(t_{k}\right), \mu_{m}\left(t_{0}\right), \mu_{p}\left(t_{k}\right), \mu_{p}\left(t_{0}\right)\right]^{T}, \Sigma_{k}\right) .
\end{aligned}
$$

$\mathrm{OD}_{\Delta}$ is then a linear transformation of these variables, $\mathrm{OD}_{\Delta}=$ $a \cdot f_{k}$, where $a$ is the column vector $a=[1,-1,-1,1](a: 1 \times 4)$. Parameter $\mathrm{OD}_{\Delta}$ is then distributed as a univariate normal distribution, $\mathrm{OD}_{\Delta} \sim N\left(a \cdot \mu_{k}, a \Sigma_{k} a^{T}\right)=N\left(\mu\left(\mathrm{OD}_{\Delta}\right), \sigma^{2}\left(\mathrm{OD}_{\Delta}\right)\right)$. Credible intervals of $\mathrm{OD}_{\Delta}$ as defined by its normal distribution were calculated to determine whether $\mathrm{OD}_{\Delta}=0$ lies within the $95 \%$ credible region. If zero was not in this region, the difference between parent and mutant strain was considered significant at this time point.

\section{Applying B-GREAT to yeast population growth data}

Population growth data for 96 yeast strains under cycloheximide exposure and PQ exposure were modeled using the stress interaction test of B-GREAT (equation 17), where $\mathrm{MM} P \mathrm{PQ}$ was substituted for $\mathrm{mM}$ cycloheximide where appropriate. The strain BY4741 was used as the control strain, and growth in yeast extract peptone dextrose (YPD) was used as the control growth condition. BF calculation and permutations of $\mathrm{BF}$ scores for each strain were performed as described above. 


\section{Testing for enrichment in significant strains}

Significance for enrichment of $S$. paradoxus strains as having significant growth phenotypes was tested using a hypergeometric distribution, using the hypergeom package from scipy.

\section{Data access}

All code and data from this study have been submitted to https ://github.com/ptonner/gp_growth_phenotype and are available in the Supplemental Methods archive. Raw growth data for $H$. salinarum used in this study are available in Supplemental Table S1.

\section{Acknowledgments}

P.D.T. was funded by a National Science Foundation (NSF) Graduate Research Fellowship. B.E.E. was funded by the National Institutes of Health (NIH) R00 HG006265, NIH R01 MH101822, and a Sloan Faculty Fellowship (Alfred P. Sloan Foundation). A.K.S. was funded by NSF MCB-141-7750. Any opinions, findings, and conclusions or recommendations expressed in this material are those of the author(s) and do not necessarily reflect the views of the National Science Foundation.

\section{References}

Baranyi J, Roberts TA. 1995. Mathematics of predictive food microbiology. Int J Food Microbiol 26: 199-218.

Bar-Joseph Z, Gitter A, Simon I. 2012. Studying and modelling dynamic biological processes using time-series gene expression data. Nat Rev Genet 13: $552-564$.

Barsa CS, Normand MD, Peleg M. 2012. On models of the temperature effect on the rate of chemical reactions and biological processes in foods. Food Eng Rev 4: 191-202.

Benavoli A, Mangili F. 2015. Gaussian processes for Bayesian hypothesis tests on regression functions. In Proceedings of the 18th international conference on artificial intelligence and statistics (AISTATS), Vol. 38, pp. 74-82. AISTATS, San Diego.

Bonneau R, Facciotti MT, Reiss DJ, Schmid AK, Pan M, Kaur A, Thorsson V, Shannon P, Johnson MH, Bare JC, et al. 2007. A predictive model for transcriptional control of physiology in a free living cell. Cell 131: 1354-1365.

Brooks AN, Reiss DJ, Allard A, Wu W, Salvanha DM, Plaisier CL, Chandrasekaran S, Pan M, Kaur A, Baliga NS. 2014. A system-level model for the microbial regulatory genome. Mol Syst Biol 10: 740 .

Cao R, Francisco-Fernández M, Quinto EJ. 2010. A random effect multiplicative heteroscedastic model for bacterial growth. BMC Bioinformatics 11: 77.

Deregowska A, Skoneczny M, Adamczyk J, Kwiatkowska A, Rawska E Skoneczna A, Lewinska A, Wnuk M. 2015. Genome-wide array-CGH analysis reveals $Y R F 1$ gene copy number variation that modulates genetic stability in distillery yeasts. Oncotarget 6: 30650-30663.

di Sciascio F, Amicarelli AN. 2008. Biomass estimation in batch biotechnological processes by Bayesian Gaussian process regression. Comput Chem Eng 32: 3264-3273.

Di Veroli GY, Fornari C, Goldlust I, Mills G, Koh SB, Bramhall JL Richards FM, Jodrell DI. 2015. An automated fitting procedure and software for dose-response curves with multiphasic features. Sci Rep 5: 14701 .

Egli T. 2009. Growth kinetics, bacterial. In Encyclopedia of microbiology, 3rd ed. (ed. Schaechter M), pp. 180-193. Academic Press, Oxford.

Fernandez-Ricaud L, Warringer J, Ericson E, Pylvninen I, Kemp GJL, Nerman O, Blomberg A. 2005. PROPHECY-a database for high-resolution phenomics. Nucleic Acids Res 33: D369-D373.

Fiebig A, Herrou J, Willett J, Crosson S. 2015. General stress signaling in the alphaproteobacteria. Annu Rev Genet 49: 603-625.

Fusi N, Listgarten J. 2016. Flexible modelling of genetic effects on functionvalued traits. In Research in computational molecular biology (ed. Singh M), pp. 95-110. Springer, Basel, Switzerland.

Gasch AP, Spellman PT, Kao CM, Carmel-Harel O, Eisen MB, Storz G, Botstein D, Brown PO. 2000. Genomic expression programs in the re- sponse of yeast cells to environmental changes. Mol Biol Cell 11: $4241-4257$.

Gommers PJF, van Schie BJ, van Dijken JP, Kuenen JG. 1988. Biochemical limits to microbial growth yields: an analysis of mixed substrate utilization. Biotechnol Bioeng 32: 86-94.

Hensman J, Lawrence ND, Rattray M. 2013. Hierarchical Bayesian modelling of gene expression time series across irregularly sampled replicates and clusters. BMC Bioinformatics 14: 1-12.

Imlay JA. 2003. Pathways of oxidative damage. Annu Rev Microbiol 57: 395-418.

Jenkins DE, Schultz JE, Matin A. 1988. Starvation-induced cross protection against heat or $\mathrm{H}_{2} \mathrm{O}_{2}$ challenge in Escherichia coli. J Bacteriol 170: 3910-3914.

Kahm M, Hasenbrink G, Lichtenberg-Frat H, Ludwig J, Kschischo M. 2010. grofit: fitting biological growth curves with R. J Stat Softw 33: $1-21$.

Kass RE, Raftery AE. 1995. Bayes factors. I Am Stat Assoc 90: 773-795.

Kaur A, Pan M, Meislin M, Facciotti MT, El-Gewely R, Baliga NS. 2006. A systems view of haloarchaeal strategies to withstand stress from transition metals. Genome Res 16: 841-854.

Kühn C, Klipp E. 2012. Zooming in on yeast osmoadaptation. Adv Exp Med Biol 736: 293-310.

Lewis NE, Nagarajan H, Palsson BO. 2012. Constraining the metabolic genotype-phenotype relationship using a phylogeny of in silico methods. Nat Rev Microbiol 10: 291-305.

Liti G, Carter DM, Moses AM, Warringer J, Parts L, James SA, Davey RP, Roberts IN, Burt A, Koufopanou V, et al. 2009. Population genomics of domestic and wild yeasts. Nature 458: $337-341$.

Lu C, Brauer MJ, Botstein D. 2009. Slow growth induces heat-shock resistance in normal and respiratory-deficient yeast. Mol Biol Cell 20: 891-903.

MacKay DJC. 1992. Bayesian interpolation. Neural Comput 4: 415-447.

Mangravite LM, Engelhardt BE, Medina MW, Smith JD, Brown CD, Chasman DI, Mecham BH, Howie B, Shim H, Naidoo D, et al. 2013. A statin-dependent QTL for GATM expression is associated with statin-induced myopathy. Nature 502: 377-380.

McKellar R, Lu X. 2003. Primary models. In Modeling microbial responses in food, 1st ed. (ed. McKellar R, Lu X), pp. 21-62. CRC Press, Boca Raton.

Millman KJ, Aivazis M. 2011. Python for scientists and engineers. Comput Sci Eng 13: 9-12.

Monod J. 1949. The growth of bacterial cultures. Annu Rev Microbiol 3: 371-394.

Neal RM. 2012. Bayesian learning for neural networks, Vol. 118. Springer Science \& Business Media, New York.

Neath AA, Cavanaugh JE. 2012. The Bayesian information criterion: background, derivation, and applications. Wiley Interdiscip Rev Comput Stat 4: $199-203$.

Ng WV, Kennedy SP, Mahairas GG, Berquist B, Pan M, Shukla HD, Lasky SR, Baliga NS, Thorsson V, Sbrogna J, et al. 2000. Genome sequence of Halobacterium species NRC-1. Proc Natl Acad Sci 97: 12176-12181.

Nichols RJ, Sen S, Choo YJ, Beltrao P, Zietek M, Chaba R, Lee S, Kazmierczak KM, Lee KJ, Wong A, et al. 2011. Phenotypic landscape of a bacterial cell. Cell 144: 143-156.

Oren A. 2008. Microbial life at high salt concentrations: phylogenetic and metabolic diversity. Saline Systems 4: 1-13.

Palacios AP, Marn JM, Quinto EJ, Wiper MP. 2014. Bayesian modeling of bacterial growth for multiple populations. Ann Appl Stat 8: $1516-1537$.

Peck RF, DasSarma S, Krebs MP. 2000. Homologous gene knockout in the archaeon Halobacterium salinarum with ura3 as a counterselectable marker. Mol Microbiol 35: 667-676.

Pedersen S, Bloch PL, Reeh S, Neidhardt FC. 1978. Patterns of protein synthesis in E. coli: a catalog of the amount of 140 individual proteins at different growth rates. Cell 14: 179-190.

Peleg M, Corradini MG. 2011. Microbial growth curves: what the models tell us and what they cannot. Crit Rev Food Sci Nutr 51: 917-945.

Plaisier CL, Lo F, Ashworth J, Brooks AN, Beer KD, Kaur A, Pan M, Reiss DJ, Facciotti MT, Baliga NS. 2014. Evolution of context dependent regulation by expansion of feast/famine regulatory proteins. BMC Syst Biol 8: 122 .

Rasmussen CE, Williams KI. 2006. Gaussian processes for machine learning. MIT Press, Cambridge, MA.

Richards FJ. 1959. A flexible growth function for empirical use. J Exp Bot 10: 290-301.

Ross T, Dalgaard P. 2003. Secondary models. In Modeling microbial responses in food, 1st ed. (ed. McKellar R, Lu X), pp. 63-150. CRC Press, Boca Raton.

Schmid AK, Reiss DJ, Pan M, Koide T, Baliga NS. 2009. A single transcription factor regulates evolutionarily diverse but functionally linked

\section{Genome Research}

www.genome.org 
metabolic pathways in response to nutrient availability. Mol Syst Biol 5: 282.

Schmid AK, Pan M, Sharma K, Baliga NS. 2011. Two transcription factors are necessary for iron homeostasis in a salt-dwelling archaeon. Nucleic Acids Res 39: 2519-2533.

Schnute J. 1981. A versatile growth model with statistically stable parameters. Can J Fish Aquat Sci 38: 1128-1140.

Sekse C, Bohlin J, Skjerve E, Vegarud GE. 2012. Growth comparison of several Escherichia coli strains exposed to various concentrations of lactoferrin using linear spline regression. Microbial Inform Exp 2: 5.

Shah A, Wilson A, Ghahramani Z. 2014. Student- $t$ processes as alternatives to Gaussian processes. In Proceedings of the 17th international conference on artificial intelligence and statistics (AISTATS), Vol. 33, pp. 877-885. AISTATS, Reykjavik, Iceland.

Sharma K, Gillum N, Boyd JL, Schmid A. 2012. The RosR transcription factor is required for gene expression dynamics in response to extreme oxidative stress in a hypersaline-adapted archaeon. BMC Genomics 13: 351.

Solak E, Murray-smith R, Leithead WE, Leith DJ, Rasmussen CE. 2003. Derivative observations in Gaussian process models of dynamic systems. In Advances in neural information processing systems (ed. Becker S, et al.), Vol. 15, pp. 1057-1064. MIT Press, Cambridge, MA.

Stephen DWS, Rivers SL, Jamieson DJ. 1995. The role of the YAP1 and YAP2 genes in the regulation of the adaptive oxidative stress responses of Saccharomyces cerevisiae. Mol Microbiol 16: 415-423.

Stephens M, Balding DJ. 2009. Bayesian statistical methods for genetic association studies. Nat Rev Genet 10: 681-690.

Tipping ME. 2001. Sparse Bayesian learning and the relevance vector machine. J Mach Learn Res 1: 211-244.

Todor H, Sharma K, Pittman AMC, Schmid AK. 2013. Protein-DNA binding dynamics predict transcriptional response to nutrients in archaea. Nucleic Acids Res 41: 8546-8558.

Todor H, Dulmage K, Gillum N, Bain JR, Muehlbauer MJ, Schmid AK. 2014. A transcription factor links growth rate and metabolism in the hypersaline adapted archaeon Halobacterium salinarum. Mol Microbiol 93: $1172-1182$.

Todor H, Gooding J, Ilkayeva OR, Schmid AK. 2015. Dynamic metabolite profiling in an archaeon connects transcriptional regulation to metabolic consequences. PLoS One 10: e0135693.
Tonner PD, Pittman AMC, Gulli JG, Sharma K, Schmid AK. 2015. A regulatory hierarchy controls the dynamic transcriptional response to extreme oxidative stress in archaea. PLoS Genet 11: e1004912.

Twarog NR, Stewart E, Hammill CV, Shelat AA. 2016. BRAID: a unifying paradigm for the analysis of combined drug action. Sci Rep 6: 25523.

Verghese J, Abrams J, Wang Y, Morano KA. 2012. Biology of the heat shock response and protein chaperones: budding yeast (Saccharomyces cerevisiae) as a model system. Microbiol Mol Biol Rev 76: 115-158.

Warringer J, Ericson E, Fernandez L, Nerman O, Blomberg A. 2003. High-resolution yeast phenomics resolves different physiological features in the saline response. Proc Natl Acad Sci 100: 15724-15729.

Yao AI, Facciotti MT. 2011. Regulatory multidimensionality of gas vesicle biogenesis in Halobacterium salinarum NRC-1. Archaea 2011: 716456.

Yoon SH, Reiss DJ, Bare JC, Tenenbaum D, Pan M, Slagel J, Moritz RL, Lim S, Hackett M, Menon AL, et al. 2011. Parallel evolution of transcriptome architecture during genome reorganization. Genome Res 21: 1892-1904.

Yoon SH, Turkarslan S, Reiss DJ, Pan M, Burn JA, Costa KC, Lie TJ, Slagel J, Moritz RL, Hackett M, et al. 2013. A systems level predictive model for global gene regulation of methanogenesis in a hydrogenotrophic methanogen. Genome Res 23: 1839-1851.

You C, Okano H, Hui S, Zhang Z, Kim M, Gunderson CW, Wang Y, Lenz P, Yan D, Hwa T. 2013. Coordination of bacterial proteome with metabolism by cyclic AMP signalling. Nature 500: 301-306.

Zhu Y, Kumar S, Menon AL, Scott RA, Adams MWW. 2013. Regulation of iron metabolism by Pyrococcus furiosus. J Bacteriol 195: 24002407.

Zuber P. 2009. Management of oxidative stress in Bacillus. Annu Rev Microbiol 63: 575-597.

Zwietering $\mathrm{MH}$, Jongenburger I, Rombouts FM, van't Riet K. 1990. Modeling of the bacterial growth curve. Appl Environ Microbiol 56: 1875-1881.

Received May 24, 2016; accepted in revised form November 15, 2016. 


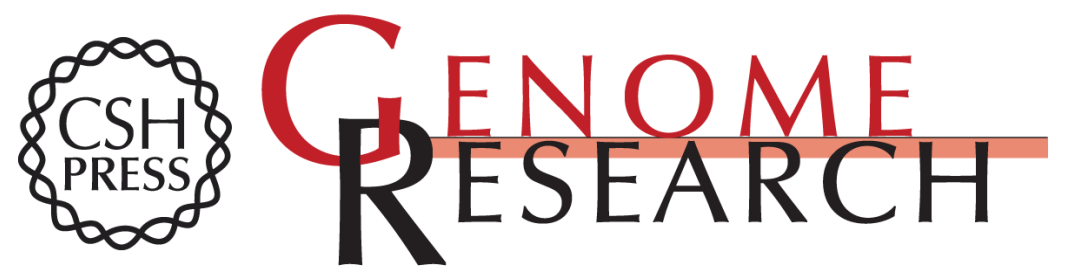

\section{Detecting differential growth of microbial populations with Gaussian process regression}

Peter D. Tonner, Cynthia L. Darnell, Barbara E. Engelhardt, et al.

Genome Res. 2017 27: 320-333 originally published online November 18, 2016

Access the most recent version at doi:10.1101/gr.210286.116

Supplemental Material

References

Open Access

Creative Commons

License

Email Alerting Service
http://genome.cshlp.org/content/suppl/2017/01/23/gr.210286.116.DC1

This article cites 60 articles, 13 of which can be accessed free at: http://genome.cshlp.org/content/27/2/320.full.html\#ref-list-1

Freely available online through the Genome Research Open Access option.

This article, published in Genome Research, is available under a Creative Commons License (Attribution 4.0 International), as described at http://creativecommons.org/licenses/by/4.0/.

Receive free email alerts when new articles cite this article - sign up in the box at the top right corner of the article or click here.

\section{Affordable, Accurate Sequencing.}

